\title{
NICHE DIVERSITY CAN EXPLAIN CROSS-CULTURAL DIFFERENCES IN PERSONALITY STRUCTURE
}

\author{
PAUL E. SMALDINO ${ }^{1, *}$, AARON LUKASZEWSKI ${ }^{2}$, CHRISTOPHER VON RUEDEN $^{3}$, \\ AND MICHAEL GURVEN ${ }^{4}$
}

April 5, 2019

\begin{abstract}
The structure of personality refers to the covariation among specific behavioral patterns in a population. Statistically derived models of personality - such as the Big Five or HEXACO models - usually assume that the covariance structure of personality characteristics is a human universal. Cross-cultural studies, however, have challenged this view, finding that less complex societies exhibit stronger covariation among behavioral characteristics, resulting in fewer derived personality factors. To explain these results, we propose the niche diversity hypothesis, which predicts that a greater diversity of social and ecological niches elicits a more diverse set of multivariate behavioral profiles, and hence lower trait covariance, at the population level. We formalize this hypothesis as a computational model in which individuals assort into niches, which influence their behavioral traits. The model provides strong support for the niche diversity hypothesis and reproduces empirical results from recent cross-cultural studies. The model also provides a novel prediction for which we find support empirically: individual trait variation increases with niche diversity. This work provides a general explanation for differences in personality structure between populations in both humans and other animals. It also suggests a radical reimagining of personality trait research: instead of reifying statistical descriptions of manifest personality structures, research should focus more attention on modeling their underlying causes.
\end{abstract}

Keywords: individual differences | social complexity | agent-based model | Big Five

\footnotetext{
${ }^{1}$ Department of Cognitive and Information Sciences, University of California, Merced

${ }^{2}$ Department of Psychology, California State University, Fullerton

${ }^{3}$ Jepson School of Leadership Studies, University of Richmond

${ }^{4}$ Department of Anthropology, University of California, Santa Barbara

E-mail address: paul.smaldino@gmail.com.
} 


\section{INTRODUCTION}

Personality refers to persistent patterns of behavior that are reasonably stable within individuals across time or contexts [1, 2, 3, 4, 5, 6, 7, 8, Within populations, specific behavioral indicators often covary, motivating researchers to reduce the personality landscape into structural models. This statistical approach organizes patterns of behavioral covariation within broad personality dimensions. For example, the Five Factor Model, or "Big Five," postulates five orthogonal, broadband dimensions - agreeableness, conscientiousness, extraversion, neuroticism, and openness to experience [6, 7, 8, 9]-each of which encompasses specific behavioral indicators that tend to loosely covary. These trait dimensions are identified inductively by factor analyzing covariance patterns among comprehensive sets of person descriptors; the resulting personality factors are frequently regarded as (i) being cross-culturally universal, and (ii) reflecting species-typical latent psychological traits (e.g., [7, 8, 10, 11) $)^{1}$.

To date, the vast majority of data collected to generate structural models of personality comes from large, modern, industrialized societies. Yet, the five factor structure has failed to replicate when tested within smaller-scale societies [12, 13, 14], in several low income country samples [15], or across a more inclusive set of natural lexicons [16]. Moreover, while many studies have looked at cultural differences in levels of specific traits (e.g. [17, 18]), few have explicitly considered how social and ecological forces shape the emergence of personality covariance structures (e.g., factors) in a population.

In recent years, a growing number of researchers have rejected the theory that inductively derived personality dimensions correspond directly to latent psychological traits, and have adopted an alternative paradigm that views patterns of personality trait covariance as emerging dynamically from many distinct psychological mechanisms and processes [19, 20, 14, 21, 4, 22, 5]. This "emergentist" perspective argues that personality trait factors reflect patterns of specific behaviors, which are influenced by other aspects of an individual's phenotype as well as by the physical and social environment [23, 24, 25, 26, 27, 28, 29, 30, 31]. From the emergentist perspective, there is no theoretical basis for expecting universal personality trait factors; in principle, many different behavioral covariance patterns could be elicited within a local ecology. For example, Nettle [32] suggests that ambition and sociability covary as part of an extraversion continuum because they tend to have synergistic effects on individuals' goal achievement. If the synergism of particular behaviors differs across societies, we need not expect a universal structure of personality covariation. Rather, the number and phenotypic content of broadband personality dimensions would be expected to vary across human populations.

One way to compare human populations is in terms of the variety of social and ecological niches available to individuals as avenues to social or material productivity. In ecological biogeography, a niche generally describes the fit of a species to particular environmental conditions [33]. Closely related organisms sharing a common niche tend to possess similar functional traits, though narrower definitions of niche describe the biotic

\footnotetext{
${ }^{1}$ An alternative HEXACO model of personality structure contains six factors that are rotated slightly differently in factor space [1. However, all of our claims about the Big Five model apply equally to the HEXACO model, and indeed to any other inductively derived covariance structures.
} 
and abiotic relationships for each species [34]. For our purposes, a niche refers to a particular way of extracting resources from the environment and/or from other individuals, and thus is situated with respect to the socioecological features of the local surroundings. Theoretically, niches define incentive structures for doing certain things or behaving in certain ways. Different niches create different payoffs to particular personality profiles. An industrialized society with advanced communication and transportation infrastructure, extensive division of labor, and high economic mobility would present many more available niches to the average person than would a small-scale foraging society in which there may be fewer opportunities for social differentiation beyond sex- and age-based division of labor [14]. Following prior research [35, 36], we refer to a culture with a greater or smaller number of such niches as more or less complex, respectively. Note that no assumptions are made about the complexity, psychological or otherwise, of individuals within each culture, or of the sophistication of particular cultural traits.

Our targets of explanation are the relatively stable distributions of adult personality traits. Some studies have indicated that children appear to exhibit more complex personality structures relative to adults [37]. Childhood is often a time of intense exploration and variation, involving broad search in the space of possible actions and outcomes [38. We focus on adult personality traits because by adulthood there is substantial canalization into roles and routines.

If individuals' personality traits are at least moderately plastic and influenced by their social and ecological niches, then the diversity of multivariate trait profiles in a population should reflect the diversity of niches. Specifically, we propose that a greater diversity of niches available in a population's local ecology will cause there to be a greater diversity of unique personality profiles, and hence a more variegated personality structure in that population. We call this the niche diversity hypothesis. The logic is that, at the individual level, a closer fit between niche and personality should result in higher payoffs, whether in the currency of performance, productivity, popularity, or reproductive fitness. Individuals are therefore incentivized to adjust their behavioral traits to the demands of their niche through a mixture of learning (e.g. via state-behavior feedbacks [39]) and assortment toward niches that provide a closer fit to their current personalities. At the population level, a greater diversity of personality profiles due to greater niche diversity should result in a population-level factor structure that is characterized by (1) lower overall trait covariance and (2) the emergence of a larger number of explanatory factors, each of which accounts for a smaller proportion of the overall variance.

The literature on non-human animals contains many examples of individual behavioral traits responding adaptively to aspects of a niche [40, 41, 42, 26, 43, including a recent study showing dramatic between-population variation in within-population trait correlations in delicate skinks [44. However, the empirical literature on niche specialization and personality in humans is rather sparse. Nevertheless, several recent studies provide preliminary evidence to support the core premises and predictions of the niche diversity hypothesis. For example, consistent with the premise that the payoffs of occupying specific niches is a function of niche-personality fit, Denissen et al. [45] reported that the match between Germans' personality profiles and the expert-rated personality demands of their occupations uniquely predicted their actual earnings. These findings 
are consistent with certain premises from social investment theory [46, 47, 48, which holds that personality trait levels may change in response to occupying specific social roles (e.g., parenthood, an occupation). However, social investment theorists have always assumed the phenotypic reality of the big five trait factors, and employed these as their units of analysis for personality change.

Two recent studies provide specific evidence for the niche diversity hypothesis, which predicts larger correlations among personality indicators in smaller-scale societies than in industrialized societies. First, Gurven et al. [14 translated the 44-item Big Five Inventory (BFI) into the language of the Tsimane, forager-horticulturalists living in the Bolivian Amazon. After one question was removed after the researchers failed to find a culturally appropriate equivalent, the inventory was administered to 632 Tsimane adults. Exploratory and confirmatory factor analyses suggested that observed personality covariation was best described by just two very broad and heterogeneous factors, tentatively labeled "prosociality" and "industriousness." Second, considering only industrialized societies studied using the same Big Five Inventory (BFI), Lukaszewski et al. 35] found larger correlations among the Big Five personality factors in less complex societies. These researchers employed a large international dataset to demonstrate that cross-national variation in the average degree of personality covariation tracks nationlevel proxies for socioecological complexity. Big Five dimensions were more strongly inter-correlated among nations of lower estimated complexity and niche diversity, even after adjusting for several potential confounders.

While this preliminary evidence for the niche diversity hypothesis is suggestive, conclusive empirical tests are hindered by the difficulty of accurately operationalizing the niche diversity of societies and by the paucity of personality data from smaller-scale societies. For example, Lukaszewski et al. [35] used product sectoral diversity, urbanization, and human development indices as their proxies of socioecological diversity. While these indirect proxies may be reasonable indicators of economic diversification, they do not include the wide range of social niches that may be available in a population. Moreover, as a verbal theory, the niche diversity hypothesis suffers from a relative lack of precision regarding specific predictions about the relationships between niche structures and population-level patterns of personality covariation. More generally, there are few other extant theories that can explicitly predict or explain the emergence of different manifest personality covariance structures across populations (for steps in this direction, see $[20,5]$.

In order to help overcome these hurdles and push toward a more precise theory of personality trait distributions and structure, we develop and analyze a simple formal model to study the relationships between niche diversity, emergent personality covariation, and factor structure. Most formal models of personality traits have either concerned traits at the individual level only [49] or focused on the persistence of multiple traits (usually two) in a population due to frequency-biased selection or payoffs to division of labor [50, 51, 52, 53, 54, 55]. Our model instead concerns the proposal that cross-cultural variation in personality trait structure can be largely explained by differences in the diversity of niches exerting an influence on individual traits. We assume only that (1) individuals possess a large number of behavioral traits that act as a source of inter-individual 
variation; (2) during development, individuals assort into socioecological niches, each of which is characterized by an ideal set of trait values that corresponds to optimal success in that niche; and (3) individuals' traits are sufficiently plastic, such that during development they partially conform to the ideal trait profiles of their niche. We will show that our model not only explains the existing empirical results, but also produces novel hypotheses for the cross-cultural study of personality at both the individual and population level.

\section{MODEL}

Consider a population of $N$ individuals. Each individual $i$ has a set of $K$ traits, $T_{i}=\left\{t_{i 1}, \ldots, t_{i K}\right\}$, where each trait is a real number in $[0,1]$. These traits should be viewed as specific behavioral or cognitive tendencies, such as those that are involved in comprising an individual's personality. An individual's trait profile (i.e. personality) can be viewed as a position in a $K$-dimensional trait-space. Initially, all traits are drawn at random from a uniform distribution $U[0,1]$. We call the initial trait profile the individual's temperament, to reflect the variation in individuals' proclivities to develop certain characteristics.

The socioecological environment consists of $M$ niches, which influence the traits of the agents that engage with them. Niches reflect social or economic roles or group settings that involve a subset of the total population, and so the number of niches, $M$, provides a simple measure of the socioecological complexity of the society. Each niche $j$ has an ideal trait profile characterized by $K$ ideal traits, $T_{j}=\left\{\tau_{i 1}, \ldots, \tau_{i K}\right\}$, where each trait is a real number in $[0,1]$. An ideal trait profile can be thought of as the unique personality type resulting in the highest payoff in that niche. Unlike the traits of individuals, the ideal traits of a niche are not mutable, but fixed. This unique set of niche traits remains fixed because it is reinforced by engaging with a particular niche, whether because the traits are useful for successful completion of tasks associated with the niche (such as persistence and critical thinking in academia), or because it is normatively reinforced through social feedback. Niche traits are drawn at random from a uniform distribution $U[0,1]$.

Dynamics. Model dynamics consist of two stages: (1) assortment, and (2) influence (Figure 1). In the assortment stage, each agent chooses to engage with a particular niche. This represents division into cultural roles, in which an individual preferentially engages with a certain set of tasks, scenarios, and social partners determined by his or her niche. Note that this assumption is valid even for cases where individuals spend much of their time outside the confines of their niche, as long as the niche represents the specific influences felt only by its members. We assume that each niche has a fixed capacity - a maximum number of individuals that can engage with it without compromising efficiency or any other functional loss. This capacity reflects the idea that the number of available niches is determined by the requirements of a society. Therefore, on the one hand, niches should not remain empty, and on the other hand, too many individuals engaging with a single niche can hinder communication and cooperation. The capacity $c$ of each niche 


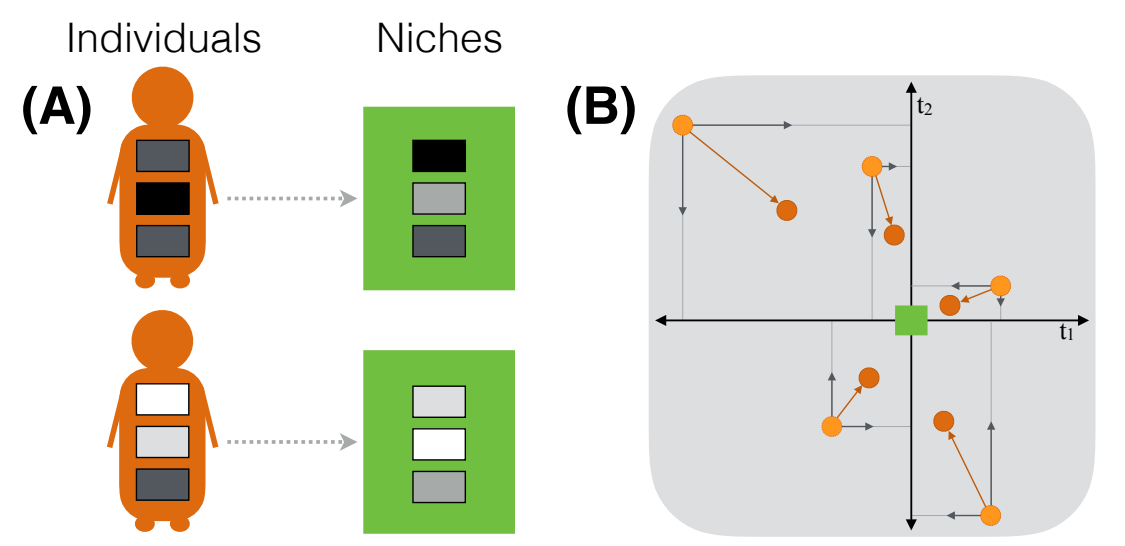

Figure 1. Illustration of the model dynamics. (A) Individuals first assort into niches that best match their trait profile. Here we see two agents and two niches, with $K=3$. Although neither agent is a perfect match with either niche, the average distance is minimized when the top agent engages with the top niche and the bottom agent engages with the bottom niche. (B) Niches influence individuals. Trait-space is represented here by a Cartesian plane centered on the niche's position $(K=2$ in this example). Five agents are represented by light orange circles, each at an initial location (temperament). Each trait value for each agent moves a proportion $p$ of the distance between its initial value and the niche's ideal value, yielding each agent a new position in trait-space represented by the darker orange circles.

is given by

$$
c=\operatorname{ceil}(N / M),
$$

where ceil(x) is the ceiling function, such that a real number $x$ is rounded up to the smallest integer greater than or equal to $x$. Thus, an environment with $M$ niches will observe the same maximal capacity for each of its $M$ niches. In order for temperament to play a role, we assume that individuals assort into niches that best fit their initial temperaments. We do this by adopting a measure of distance from models of opinion dynamics [56, 57] so that the distance $d_{i j}$ between individual $i$ and niche $j$ is

$$
d_{i j}=\frac{1}{K} \sum_{k=1}^{K}\left|t_{i k}-\tau_{j k}\right| .
$$

Each agent, in turn, evaluates each niche and chooses the niche with the shortest distance from itself that is not already at capacity. In the SI Appendix, we also consider an alternative distance metric as well as what happens when individuals choose niches at random.

After they have all assorted into niches, each individual is influenced by its niche. Here, each individual in the niche updates his or her traits toward those values that are 
most appropriate to successful participation in the niche. For each individual $i$ in niche $j$, each trait $k$ is updated as follows:

$$
t_{i k} \leftarrow t_{i k}+p\left(\tau_{j k}-t_{i k}\right)
$$

where $p$ is the plasticity available to each individual to adjust their traits. The mathematical form of the influence rule is drawn from psychological theories of reinforcement learning [58, 59], and is often implemented in computational models of social influence [56, 60, 61, 57]. A plasticity of $p=1$ permits individuals to perfectly alter their traits to match their niche, while a plasticity of $p=0$ would disallow any updating whatsoever (implying fixed, genetically determined trait distributions). Intermediate values of $p$ reflect both some inertia imparted by individuals' temperaments as well as the malleability of traits and characteristics that occur through the process of development.

Outcome measures. We consider the extent to which greater niche diversity reduces the constraints on trait development by assessing three outcomes from our simulations. First, we consider the average inter-trait correlations, as used by [35]. That is, at the end of each simulation, we take the squared Pearson correlation between every pair of traits, and report the average of this correlation for the entire population. Second, we consider the mean trait variance at the end of each simulation, with the general prediction that higher niche diversity should lead to greater variance in personality traits. Third, we performed exploratory factor analysis on our simulation results, because we were interested in how many separate "factors" comprising bundled combinations of traits may be meaningfully characterized in different simulated societies, in order to compare our results with empirical cross-cultural variation [14].

Java code used to generate our data, SAS scripts used to perform the exploratory factor analysis, and $\mathrm{R}$ scripts used to generate our plots are all available at https: //osf.io/pyhq7/

\section{RESULTS}

We find that increased niche diversity, $M$, reduces correlations between traits (Figure 2). This is strongly moderated by plasticity, $p$, with higher plasticity producing higher correlations, especially for low $M$. At high $M$, plasticity becomes less critical for finding an appropriate niche. This result is consistent with observed population differences in personality trait covariation seen in country-level data for industrialized nations [35]. We note that lower $p$ is most consistent with the inter-trait correlations observed in those data.

We also find that with increased niche diversity comes more general variation in trait values (Figure 3). We measured the average variance in trait values as a function of plasticity, $p$, and the number of niches, $M$. We find a strong positive relationship between niche number and trait variance. When plasticity is low, the influence of $M$ is muted, as more of the initial random variation is preserved. The non-monotonic relationship between trait variance and $p$ (Figure $3 \mathrm{~B}$ )is discussed further in the SI Appendix.

That greater niche diversity should lead to more trait variance is a novel prediction derived from our analysis. We test this prediction using the same 55-nation sample 


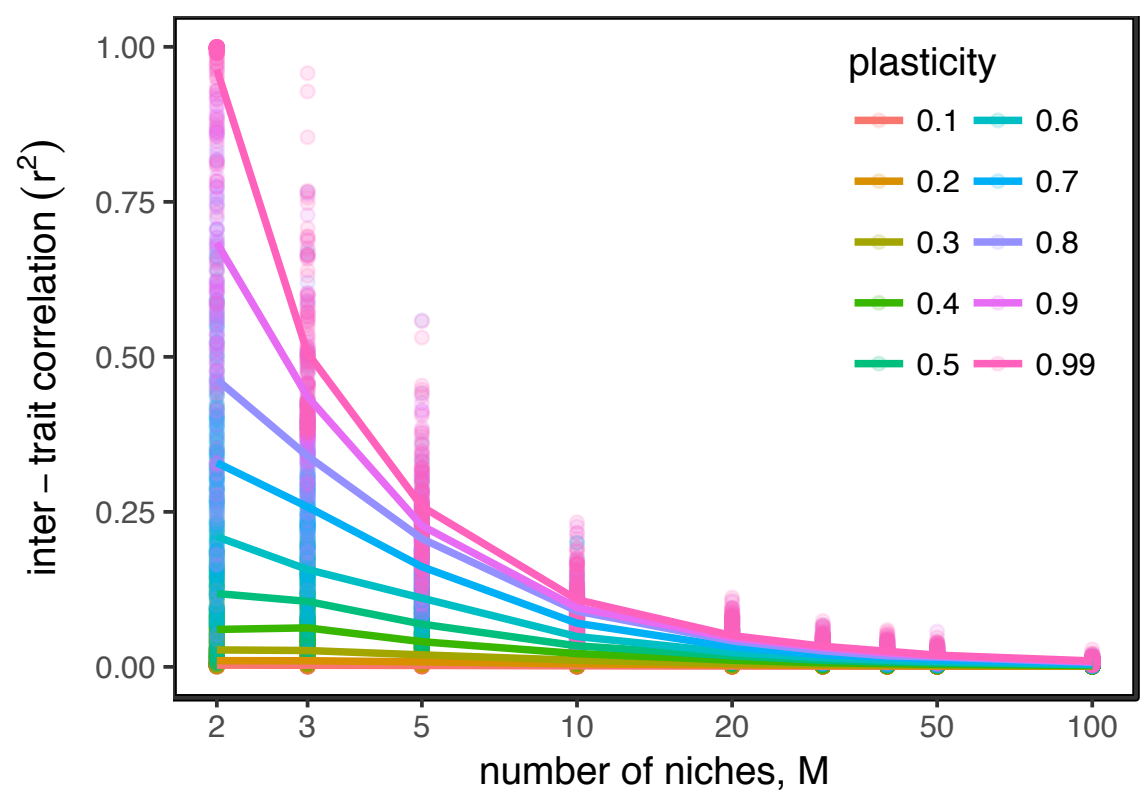

FigURE 2. Inter-trait correlation plotted as a function of niche number, $M$, for varying values of plasticity, $p$ (note the x-axis is log-scaled). Correlations decrease with the number of niches. This effect is exacerbated by increased plasticity, which allows for large correlations at low $M$. Open circles are individual model runs, solid lines are means. Data are from 100 simulation runs for each parameter combination. Here $N=1000$ and $K=5$.

of [35]. Nations scoring higher in a proxy of "socioecological complexity" (an index derived from principal components analysis of urbanization, human development index and product sectoral diversity; see [35]), show higher levels of personality trait variance (std. $\beta=0.431, p<0.001$; Figure 4). This relationship holds even after adjusting for potential confounders, including sample size, whether the BFI was conducted in the native language, two measures of scale use biases (acquiescence bias, negative item bias), and an indicator of socially desirable responding (agreeableness) (Table 1). Accounting for continent of residence in mixed models does not substantively alter these results. See SI Appendix for more details.

For our theory to be robustly applicable to personality research, it must also explain the finding that factor analysis on a fixed number of traits yields fewer explanatory factors in less complex societies [14]. We performed exploratory factor analysis (EFA) with varimax rotation across sets of 30 simulation runs, each set varying in combinations of $M$ and $p$. We kept the number of traits fixed at $K=50$, similar to the number of items in the BFI. Figure $5 \mathrm{~A}$ plots the number of factors with eigenvalues greater than 1 as a function of the number of niches, $M$, for $p=0.9$. This is a common (though imperfect) heuristic in EFA for counting factors. We find that the number of explanatory factors 

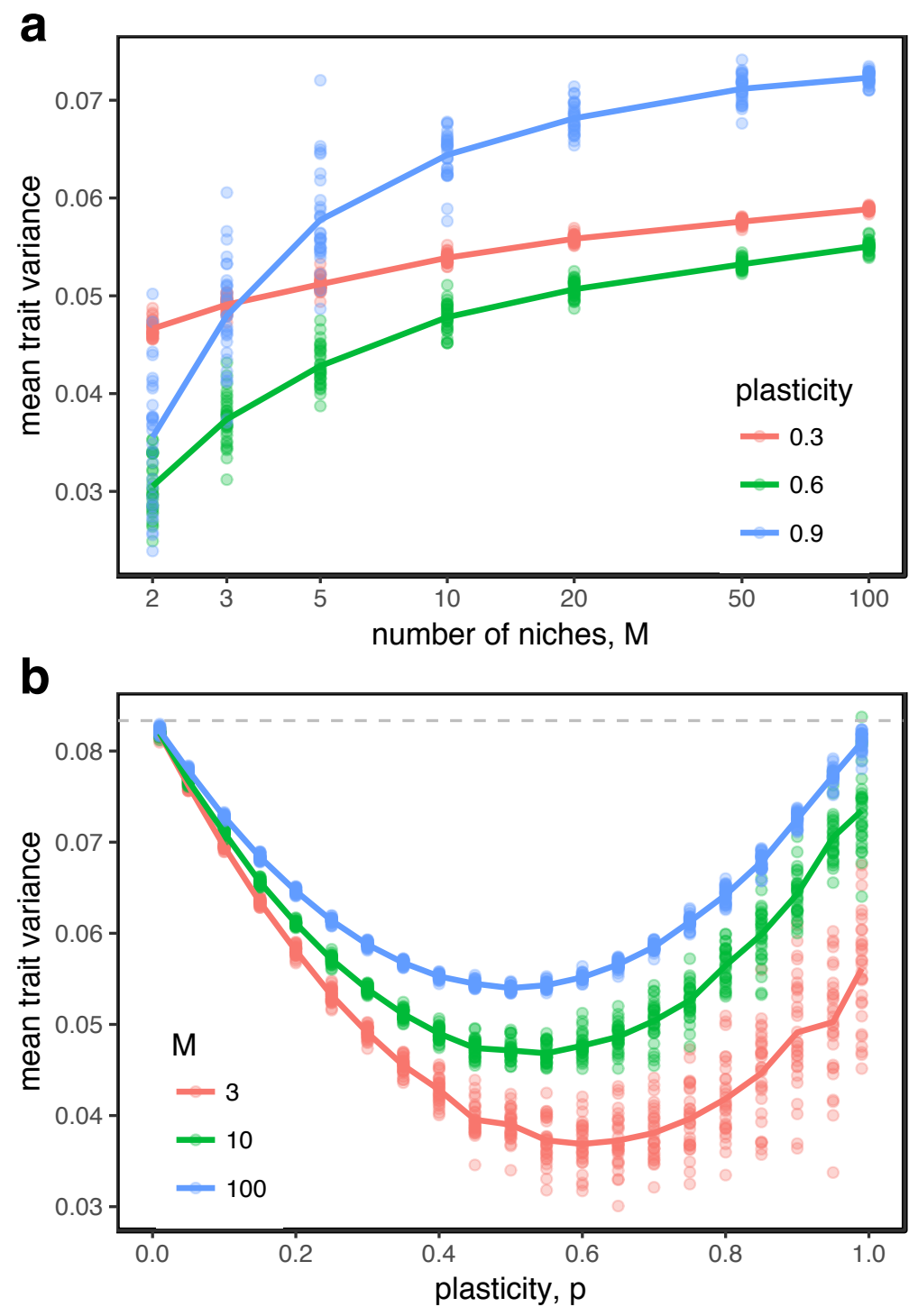

FiguRE 3. The mean variance of each trait value plotted as a function of (a) niche number, $M$, for varying values of plasticity, $p$, and (b) plasticity, $p$, for varying numbers of niches, $M$. Data are from 30 simulation runs for each parameter combination. Here $N=1000$ and $K=50$. The dashed gray line in (b) is at $1 / 12$, the expected variance of a uniform distribution in $[0,1]$. Trait variance in the model is therefore closest to random when niches either exert no influence or exert such a strong influence that the distribution of individuals' trait values approaches the random distribution of niche ideal values. 


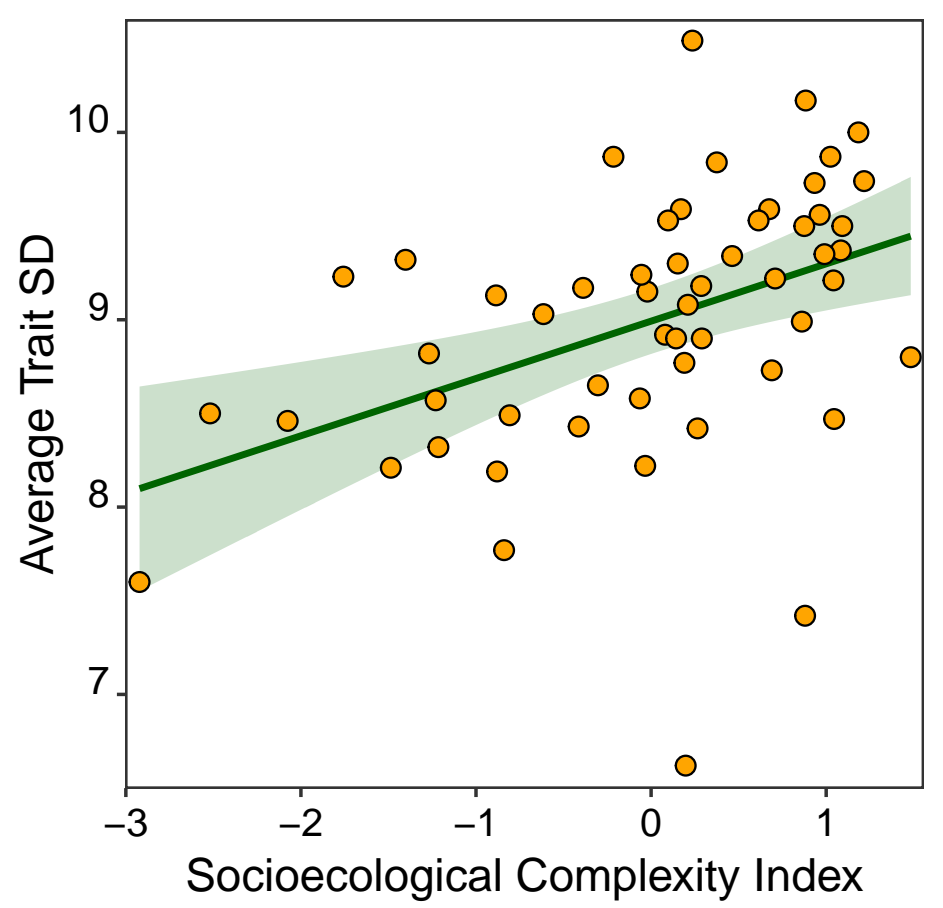

Figure 4. Average SD among each of the five factors as measured by the BFI for 55 nations plotted against each nation's index of socioecological complexity (a proxy for niche diversity). Green line is best-fit linear regression, shaded region is $95 \%$ confidence interval. Std. $\beta=0.431$, $p<0.001$.

\begin{tabular}{cccccc}
\hline \hline Variable & $\begin{array}{l}\text { Parameter Std error } \\
\text { estimate }\end{array}$ & $t$ value & Pr $>|t|$ & $\begin{array}{c}\text { Std } \\
\text { estimate } \\
(\beta)\end{array}$ \\
\hline $\begin{array}{c}\text { Socioecological } \\
\text { Complexity }\end{array}$ & 0.311 & 0.111 & 2.79 & 0.0075 & 0.438 \\
$\quad$ Index & & & & & \\
Total sample size & 0.000 & 0.000 & 0.62 & 0.5353 & 0.075 \\
BFI Language & -0.145 & 0.230 & -0.63 & 0.5331 & -0.079 \\
Acquiescence Bias & 0.070 & 0.023 & 3.02 & 0.0040 & 0.354 \\
Negative Item Bias & -0.257 & 0.128 & -2.00 & 0.0509 & -0.271 \\
Agreeableness & 0.051 & 0.031 & 1.63 & 0.1093 & 0.196 \\
$\quad$ Intercept & 3.826 & 1.815 & 2.11 & 0.0403 & 0.000 \\
\hline
\end{tabular}

TABLE 1. Multivariate regression with trait SD vs. socioecological complexity, with additional predictors. Model $R^{2}=0.4157$, Adj $R^{2}=0.3426$; $F=5.69, d f=6, p<0.0002$ 

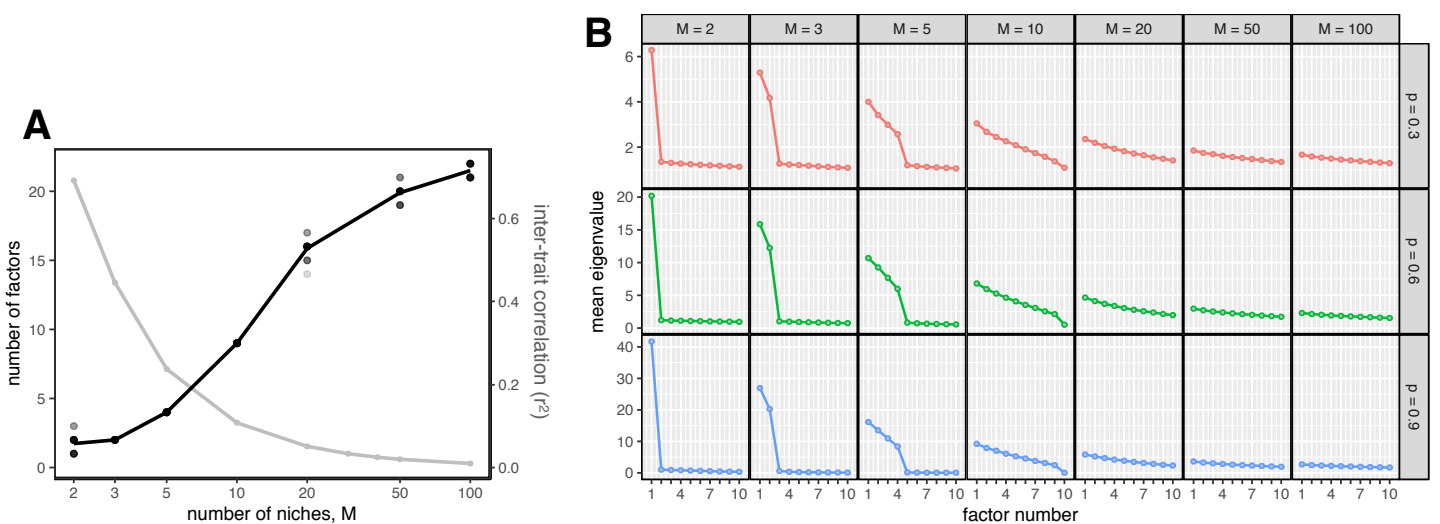

FiguRE 5. (A) The number of factors with eigenvalues greater than one plotted as a function of niche number, $M$, for $p=0.9$ (x-axis is logscaled). Circles are individual runs, the black line connects the means. The gray line is the mean inter-trait correlation for $p=0.9$, taken from Figure 2. (B) Scree plots showing the mean eigenvalues from exploratory factor analysis for the first ten factors, for varying values of $p$ and $M$. Data in both plots are from 30 simulation runs for each parameter combination, $N=1000, K=50$.

increases with the number of niches, and also illustrate how the number of factors varies inversely with the average inter-trait correlation. As the number of niches increases, the variance explained by any particular factor diminishes precipitously, as observed in the scree plots shown for a range of $M$ and $p$ values (Figure 5B). For lower values of plasticity, less variance is explained by each factor, but the number of emergent factors appears insensitive to plasticity.

Our results are robust to variation in the number of traits (Figure S2) and population size (Figure S3). Although the ability of agents to initially assort into their best-fitting niche increases inter-trait correlations for low levels of plasticity, it is not necessary to produce our main result that inter-trait correlation decreases as a function of the number of niches. Random initial assortment to niches irrespective of temperament shows a similar inverse relationship between inter-trait correlation and number of niches (Figure S4).

\section{DisCUSSION}

By focusing on the socioecological causes of behavioral variation, the niche diversity hypothesis helps explain observed patterns of personality trait variation across populations and cultures. Our simulation model in which agents adjust personality traits to suit their nearest niche is very simple, but illustrates how such sorting can result in alteration of the number and structure of personality types at the population level. With such simple rules in place, our model shows how increasing the number of niches in a population environment can reduce the correlations between personality attributes, 
and in turn increase the number of emergent factors required to explain patterns of behavioral covariance. These simulation results expand the scope of existing cross-cultural studies that focus on explaining variation in personality trait values [13, 14, 15, 16, 35, to address broader questions concerning cross-cultural variability in personality structure. In addition, niche diversity potentially offers a consilient way in which to think about disparate descriptors of societal complexity. For example, Gurven [62] recently showed that higher national-level personality trait covariation is also associated with greater cultural tightness [63, lower individualism [64], and lower gender egalitarianism [65], all of which may reflect stricter constraints limiting the availability of diverse socioecological niches to individuals within those societies.

Our analysis yields novel questions for both future modeling efforts and empirical research. First, we predict that the variance in personality trait values should be higher in more complex societies with higher niche diversity. Our preliminary analysis of one of the largest cross-cultural datasets supports this prediction. In addition, a previous study based on a different cross-national dataset found that various economic performance indicators also positively associate with variance in personality trait values [66]. Nevertheless, a full investigation of this prediction may require the collection of new empirical: both systematic comparative data assessing niches and personality, and longitudinal data assessing changes in personality structure in relation to changes in niche number and diversity. Second, we should ask more precise questions about the plasticity of the behavioral traits that compose what we usually think of as personality characteristics. There is strong evidence that personality factors are at least somewhat plastic, supported by both age-related changes [67] and those resulting from traumatic or otherwise meaningful events [68], and more broadly by the logic that, because personality traits quantify patterns of behavior, their manifestation must be influenced by the affordances provided by the physical and social environment [29, 27, 25, 26, 31, 28, 47, 48. However, most prior studies of personality plasticity have assumed the phenotypic reality of personality factors such as the Big Five and HEXACO traits (e.g., [46, 47, 48]). Our analysis implies that, if we are to understand how the environment shapes personality covariance, we must ask about the plasticity of individual behavioral traits, rather than composite factors based on patterns of behavioral covariance observed in societies of similar niche diversity.

Given these considerations, we are forced to reconsider the universality of the Big Five model of personality traits. Why does personality research regularly obtain five factors? Is the Big Five simply a coincidence of the large niche diversity found throughout largescale, urban societies? Will we obtain more factors if populations become even more complex? Or is there some other force that is restraining the number of factors? Perhaps certain suites of psychological mechanisms really are intrinsically coordinated in ways that create particular covariance patterns, which would manifest as universal factors (c.f. [11]). Whatever the answers are, we must further reconcile them with the fact that personality traits are entangled with their linguistic categories [69]. Personality isn't just clustering of behavioral traits, it's about how useful, locally-salient descriptive labels aid behavioral prediction [70, 71]. Indeed, several small-scale populations lack in their lexicon the broad range of personality descriptors so prevalent in languages commonly 
spoken in large-scale societies [16]. Niche diversity may reflect what is relevant to people in a society, and so our labels may end up being more or less diverse based on how we need to describe people [36]. It is therefore possible that personality instruments developed in the West are failing to capture some of the important variation. This is a problem that can only be solved by the development of richer, culturally-sensitive instruments. Meanwhile, we note that the analyses by Lukaszewski et al. [35] did include controls for linguistic differences (as well as for psychometric response biases), which provide evidence that language use neither explains inter-factor covariance as measured by the BFI nor alters the relationship between inter-factor covariance and socioecological complexity.

We have proposed one mechanism to explain the observed association between behavioral trait diversity and niche diversity, based on assortment and plasticity. However, evolutionary biologists have long been aware of another mechanism. If niches are inherited through assortment or limited dispersal, so that parents and offspring have similar niches, then natural selection can stabilize phenotypic variation at the genetic level. Evolutionary ecologists have provided support for the proposal that increased phenotypic variation, at the species level, will be associated with increased diversity of ecological niches [41, 72]. Nevertheless, human psychology is highly plastic, our social structure is malleable, our intra-population genetic variation is high, and our cultures vary tremendously. We therefore doubt that variation across populations in personality structure is primarily caused by the maintenance of heritable variance in specific patterns of trait covariance.

Another alternative hypothesis is based on the possibility that populations might not vary substantially in their number of socioecological niches, but only in the character of those niches. That is, cultures might vary in the extent to which individuals' behavioral traits are shaped by their socioecological niches. We might call this the plasticity hypothesis. In more complex societies, there might be relatively low plasticity, because individuals have more relative freedom to express their initial temperaments. In simpler societies, behavioral variation may be more constrained by strict social norms. For example, in some small scale societies it has been noted that certain emotional expressions are proscribed, such as proscriptions against crying among the Ache and against anger among the Matsigenka [73]. Our simulations indicate that differences in plasticity would be sufficient to lower the average inter-trait correlations in a way similar to variation in niche diversity, as seen in Figure 2. However, if variation in plasticity were driving cross-cultural differences, exploratory factor analysis would not uncover more factors as plasticity decreased, as indicated by the robustness to plasticity seen in the scree plots in Figure 5. Additionally, our empirical analysis reveals a monotonicallyincreasing relationship between trait variance and niche diversity, which supports the linear relationship predicted by the niche diversity hypothesis and not the curvilinear relationship predicted by the plasticity hypothesis (Figure 3). Thus, while variation in plasticity may indeed be an important part of explaining cross-cultural variation in personality structure, its influence is not sufficient to replace the niche diversity hypothesis. Moreover, even if existing measures do not capture all the relevant socioecological niche diversity in a population (and they surely do not), there are still good reasons to believe that such variation exists and is important [35, 36]. 
Our model used very simplified representations of niches and their influence on individuals' traits. Such simplification is necessary to characterize the behavior of a complex system as a formal model, providing a critical scaffolding for the development of richer theories about the emergence of personalities [74, 75]. In real life, individuals may simultaneously engage with multiple niches, may change niches over the course of their life, may be influenced directly by individuals both in terms of conformity (as implied by our model) and in terms of differentiation, and may be constrained in their choice of niche by socioeconomic and demographic factors. These considerations complicate the hypothesis, but do not damage it. For one thing, these considerations remain consistent with the general idea that some populations are characterized by more socioecological niches, and this variation in niche diversity influences the degree of covariation among behavioral traits in a population. For another, endless consideration of nuance can harm theory development [76], and it has been increasingly acknowledged that more formal theory is sorely needed in the behavioral sciences [77]. Nevertheless, it is important to examine the assumptions made by such a formalization, such that future work can deepen the conclusions we make and assess the robustness of our claims.

First, we represented a niche by a fixed set of trait values toward which individuals are influenced. However, people are also social creatures, and will be influenced by each other, both positively, becoming more similar, as well as differentiating themselves due to a desire to distinguish themselves from others [78, 79]. That said, our model's assumption that all participants in a niche are similarly influenced could also be viewed as a sort of conformist social influence, and so the phenotypic plasticity featured in the model could reflect social learning as much as asocial learning.

Second, we represented individuals as each belonging to a single niche, for which joining is an individual decision and in which they remain forever. In reality, individuals are drawn to or excluded from multiple niches based on their social networks, lineage, and status [80, 81]. They may participate in multiple niches simultaneously and over time [36]. More broadly, we do not model the developmental process of niche adaptation in detail, nor consider dynamics where individuals alter their niches. Future work could incorporate a more complex representation of niche structure and social influence in order to tease out how different components influence the nature of trait variation, as well as a more nuanced consideration of development (e.g., [82, 83, 84]). That said, our model is simple but captures what we believe is the quintessential feature of the niche diversity hypothesis.

Finally, the model does not deal with social and cultural change (i.e., changes in the number and complexity of niches), nor with the genetic foundations of personality variation. Instead, we assumed a fixed number of niches with fixed size. In other words, niche diversity was treated as an exogenous variable. Over generational time, the distribution of traits may influence the available niches, creating a feedback loop [39]. If we are interested in how cultural evolution interacts with trait distributions, future models will have to incorporate this feedback and consider evolutionary dynamics. Both new theoretical work and longitudinal empirical studies of personality structure in populations undergoing socioeconomic change will be necessary to further elaborate and test the niche diversity hypothesis. 
The biology of inheritance enforces some intrinsic variation among individuals. However, social and ecological forces also shape and constrain behavior and the cognitive schemas and proclivities that produce it [85, 86]. The logical consequence of this premise is that the structure of behavioral trait covariation - the essence of personality - should reflect the structure of the socioecological environment.

\section{ACKNOWLEDGMENTs}

We thank audiences at UC Merced and MPI-EVA for discussion of these ideas, and Bret Beheim, John Bunce, Joanne Chung, Eiko Fried, Anne Kandler, Dieter Lukas, Julia Rohrer, Brent Roberts, and Matt Turner for helpful comments on earlier drafts of this manuscript.

\section{REFERENCES}

[1] Ashton, M. C. \& Lee, K. Empirical, theoretical, and practical advantages of the HEXACO model of personality structure. Personality and Social Psychology Review 11, 150-166 (2007).

[2] Buss, D. M. \& Craik, K. H. The act frequency approach to personality. Psychological Review 90, 105 (1983).

[3] Fleeson, W. Toward a structure-and process-integrated view of personality: Traits as density distributions of states. Journal of Personality and Social Psychology 80, 1011 (2001).

[4] Mischel, W. \& Shoda, Y. A cognitive-affective system theory of personality: reconceptualizing situations, dispositions, dynamics, and invariance in personality structure. Psychological Review 102, 246 (1995).

[5] Wood, D. \& Denissen, J. J. A functional perspective on personality trait development. Psychology of change: Life contexts, experiences, and identities 97-115 (2015).

[6] John, O. P., Naumann, L. P. \& Soto, C. J. Paradigm shift to the integrative big five trait taxonomy. Handbook of personality: Theory and research 3, 114-158 (2008).

[7] McCrae, R. R. \& Costa Jr, P. T. Personality trait structure as a human universal. American Psychologist 52, 509 (1997).

[8] McCrae, R. R. \& Costa, P. T. Empirical and theoretical status of the five-factor model of personality traits. The SAGE handbook of personality theory and assessment 1, 273-294 (2008).

[9] Goldberg, L. R. The development of markers for the big-five factor structure. Psychological Assessment 4, 26 (1992).

[10] Bouchard, T. J. \& Loehlin, J. C. Genes, evolution, and personality. Behavior Genetics 31, 243-273 (2001).

[11] Nettle, D. Personality: What makes you the way you are (Oxford University Press, 2009).

[12] Alvergne, A., Jokela, M. \& Lummaa, V. Personality and reproductive success in a high-fertility human population. Proceedings of the National Academy of Sciences 107, 11745-11750 (2010).

[13] Bailey, D. H. et al. Heritability and fitness correlates of personality in the Ache, a natural-fertility population in paraguay. PLOS ONE 8, e59325 (2013).

[14] Gurven, M., von Rueden, C., Massenkoff, M., Kaplan, H. \& Lero Vie, M. How universal is the big five? testing the five-factor of personality variation among forager-farmers in the bolivian amazon. Journal of Personality and Social Psychology 104, 354-370 (2013).

[15] Schmitt, D. P., Allik, J., McCrae, R. R., Benet-Martinez, V. \& Ault, L. The geographic distribution of the big five personality traits: Patterns and profiles of human self-description across 56 nations. Journal of Cross-Cultural Psychology 38, 173-212 (2007).

[16] Saucier, G. et al. A basic bivariate structure of personality attributes evident across nine languages. Journal of Personality 82, 1-14 (2014).

[17] Schaller, M. \& Murray, D. R. Pathogens, personality, and culture: disease prevalence predicts worldwide variability in sociosexuality, extraversion, and openness to experience. Journal of Personality and Social Psychology 95, 212 (2008). 
[18] Schmitt, D. P., Realo, A., Voracek, M. \& Allik, J. Why can't a man be more like a woman? sex differences in big five personality traits across 55 cultures. Journal of Personality and Social Psychology 94, 168 (2008).

[19] Baumert, A. et al. Integrating personality structure, personality process, and personality development. European Journal of Personality 31, 503-528 (2017).

[20] Cramer, A. O. et al. Dimensions of normal personality as networks in search of equilibrium: You can't like parties if you don't like people. European Journal of Personality 26, 414-431 (2012).

[21] Lukaszewski, A. W. Testing an adaptationist theory of trait covariation: Relative bargaining power as a common calibrator of an interpersonal syndrome. European Journal of Personality 27, 328-345 (2013).

[22] Uher, J. Personality psychology: Lexical approaches, assessment methods, and trait concepts reveal only half of the story? Why it is time for a paradigm shift. Integrative Psychological and Behavioral Science 47, 1-55 (2013).

[23] Buss, D. M. Selection, evocation, and manipulation. Journal of Personality and Social Psychology 53, 1214-1221 (1987).

[24] De Vries, R. E., Tybur, J. M., Pollet, T. V. \& van Vugt, M. Evolution, situational affordances, and the hexaco model of personality. Evolution and Human Behavior 37, 407-421 (2016).

[25] Denissen, J. J. \& Penke, L. Motivational individual reaction norms underlying the five-factor model of personality: First steps towards a theory-based conceptual framework. Journal of Research in Personality 42, 1285-1302 (2008).

[26] Montiglio, P.-O., Ferrari, C. \& Réale, D. Social niche specialization under constraints: personality, social interactions and environmental heterogeneity. Philosophical Transactions of the Royal Society B: Biological Sciences 368, 20120343 (2013).

[27] Kagan, J. Temperamental contributions to the development of psychological profiles: I. basic issues. In Social Anxiety (Third Edition), 377-418 (Elsevier, 2014).

[28] von Rueden, C. R., Lukaszewski, A. W. \& Gurven, M. Adaptive personality calibration in a human society: effects of embodied capital on prosocial traits. Behavioral Ecology 26, 1071-1082 (2015).

[29] Jokela, M., Pekkarinen, T., Sarvimäki, M., Terviö, M. \& Uusitalo, R. Secular rise in economically valuable personality traits. Proceedings of the National Academy of Sciences 114, 6527-6532 (2017).

[30] Quinlan, R. J., Dira, S. J., Caudell, M. \& Quinlan, M. Culture and psychological responses to environmental shocks. Current Anthropology 57, 632-652 (2016).

[31] Sng, O., Neuberg, S. L., Varnum, M. E. \& Kenrick, D. T. The behavioral ecology of cultural psychological variation. Psychological review (2018).

[32] Nettle, D. Evolutionary perspectives on the five-factor model of personality. In Buss, D. M. \& Hawley, P. H. (eds.) The evolution of personality and individual differences, 5-28 (Oxford University Press Oxford, UK, 2011).

[33] Schoener, T. Ecological niche. In Levin, S. A. et al. (eds.) The Princeton guide to ecology, 3-13 (Princeton University Press, 2009).

[34] Pocheville, A. The ecological niche: History and recent controversies. In Heams, T., Huneman, P. \& Lecointre, G. (eds.) Handbook of evolutionary thinking in the sciences, 547-586 (Springer, 2015).

[35] Lukaszewski, A. W., Gurven, M., von Rueden, C. R. \& Schmitt, D. P. What explains personality covariation? A test of the socioecological complexity hypothesis. Social Psychological and Personality Science 8, 943-952 (2017).

[36] Smaldino, P. E. Social identity and cooperation in cultural evolution. Behavioural Processes 161, 108-116 (2019).

[37] Halverson, C. F. et al. Personality structure as derived from parental ratings of free descriptions of children: The inventory of child individual differences. Journal of Personality 71, 995-1026 (2003).

[38] Gopnik, A., Griffiths, T. L. \& Lucas, C. G. When younger learners can be better (or at least more open-minded) than older ones. Current Directions in Psychological Science 24, 87-92 (2015).

[39] Sih, A. et al. Animal personality and state-behaviour feedbacks: a review and guide for empiricists. Trends in Ecology 86 Evolution 30, 50-60 (2015). 
[40] Bergmüller, R. \& Taborsky, M. Animal personality due to social niche specialisation. Trends in Ecology E Evolution 25, 504-511 (2010).

[41] Araújo, M. S., Bolnick, D. I. \& Layman, C. A. The ecological causes of individual specialisation. Ecology Letters 14, 948-958 (2011).

[42] Webster, M. M. \& Ward, A. J. Personality and social context. Biological Reviews 86, 759-773 (2011).

[43] Stamps, J. \& Groothuis, T. G. The development of animal personality: relevance, concepts and perspectives. Biological Reviews 85, 301-325 (2010).

[44] Michelangeli, M., Chapple, D. G., Goulet, C. T., Bertram, M. G. \& Wong, B. Behavioral syndromes vary among geographically distinct populations in a reptile. Behavioral Ecology (2018).

[45] Denissen, J. J. et al. Uncovering the power of personality to shape income. Psychological Science 29, 3-13 (2018).

[46] Bleidorn, W. et al. Personality maturation around the world: A cross-cultural examination of socialinvestment theory. Psychological Science 24, 2530-2540 (2013).

[47] Hudson, N. W., Roberts, B. W. \& Lodi-Smith, J. Personality trait development and social investment in work. Journal of Research in Personality 46, 334-344 (2012).

[48] Roberts, B. W., Wood, D. \& Smith, J. L. Evaluating five factor theory and social investment perspectives on personality trait development. Journal of Research in Personality 39, 166-184 (2005).

[49] Nowak, A., Vallacher, R. R. \& Zochowski, M. The emergence of personality: Dynamic foundations of individual variation. Developmental Review 25, 351-385 (2005).

[50] McElreath, R. \& Strimling, P. How noisy information and individual asymmetries can make ?personality?an adaptation: a simple model. Animal Behaviour 72, 1135-1139 (2006).

[51] Dubois, F., Giraldeau, L.-A. \& Réale, D. Frequency-dependent payoffs and sequential decisionmaking favour consistent tactic use. Proceedings of the Royal Society of London B: Biological Sciences 279, 1977-1985 (2012).

[52] Lake, M. W. \& Crema, E. R. The cultural evolution of adaptive-trait diversity when resources are uncertain and finite. Advances in Complex Systems 15, 1150013 (2012).

[53] Vásárhelyi, Z., Meszéna, G. \& Scheuring, I. Evolution of heritable behavioural differences in a model of social division of labour. PeerJ 3, e977 (2015).

[54] Wolf, M., Van Doorn, G. S., Leimar, O. \& Weissing, F. J. Life-history trade-offs favour the evolution of animal personalities. Nature 447, 581 (2007).

[55] Wolf, M. \& McNamara, J. M. On the evolution of personalities via frequency-dependent selection. The American Naturalist 179, 679-692 (2012).

[56] Flache, A. \& Macy, M. W. Small worlds and cultural polarization. Journal of Mathematical Sociology 35, 146-176 (2011).

[57] Turner, M. A. \& Smaldino, P. E. Paths to polarization: How extreme views, miscommunication, and random chance drive opinion dynamics. Complexity 2018, 2740959 (2018).

[58] Rescorla, R. A. \& Wagner, A. R. A theory of Pavlovian conditioning. In Black, A. H. \& Prokasy, W. F. (eds.) Classical conditioning II: Current research and theory, 64-99 (Appleton-Century-Crofts, New York, 1972).

[59] Sutton, R. S. \& Barto, A. G. Reinforcement Learning (MIT Press, Cambridge, MA, 1998).

[60] Epstein, J. M. Agent_Zero: Toward neurocognitive foundations for generative social science (Princeton University Press, Princeton, NJ, 2014).

[61] Smaldino, P. E. \& Epstein, J. M. Social conformity despite individual preferences for distinctiveness. Royal Society Open Science 2, 140437 (2015).

[62] Gurven, M. D. Broadening horizons: Sample diversity and socioecological theory are essential to the future of psychological science. Proceedings of the National Academy of Sciences 115, 11420-11427 (2018).

[63] Gelfand, M. J. et al. Differences between tight and loose cultures: A 33-nation study. Science 332, 1100-1104 (2011). 
[64] Hofstede, G. Cultures and organizations: Software of the mind (McGraw-Hill, London, 1991).

[65] House, R. J., Hanges, P. J., Javidan, M., Dorfman, P. W. \& Gupta, V. Culture, leadership, and organizations: The GLOBE study of 62 societies (Sage Publications, Thousand Oaks, CA, 2004).

[66] Bartram, D. Scalar equivalence of OPQ32: Big Five profiles of 31 countries. Journal of CrossCultural Psychology 44, 61-83 (2013).

[67] Caspi, A., Roberts, B. W. \& Shiner, R. L. Personality development: Stability and change. Annu. Rev. Psychol. 56, 453-484 (2005).

[68] MacLean, K. A., Johnson, M. W. \& Griffiths, R. R. Mystical experiences occasioned by the hallucinogen psilocybin lead to increases in the personality domain of openness. Journal of Psychopharmacology 25, 1453-1461 (2011).

[69] Contreras Kallens, P. A., Dale, R. \& Smaldino, P. E. Cultural evolution of categorization. Cognitive Systems Research 52, 765-774 (2018).

[70] Buss, D. M. Personality and the adaptive landscape: The role of individual differences in creating and solving social adaptive problems. In Buss, D. M. \& Hawley, P. H. (eds.) The evolution of personality and individual differences, 29-57 (Oxford University Press, 2011).

[71] Wood, D. Testing the lexical hypothesis: Are socially important traits more densely reflected in the English lexicon? Journal of Personality and Social Psychology 108, 317-335 (2015).

[72] Van Valen, L. Morphological variation and width of ecological niche. The American Naturalist 99, 377-390 (1965).

[73] Hill, K. \& Hurtado, A. M. Aché life history: The ecology and demography of a foraging people (Routledge, 2017).

[74] Haldane, J. B. S. A defense of beanbag genetics. Perspectives in Biology and Medicine 7, 343-360 (1964).

[75] Smaldino, P. E. Models are stupid, and we need more of them. In Vallacher, R. R., Read, S. J. \& Nowak, A. (eds.) Computational social psychology, 311-331 (Routledge, 2017).

[76] Healy, K. Fuck nuance. Sociological Theory 35, 118-127 (2017).

[77] Muthukrishna, M. \& Henrich, J. A problem in theory. Nature Human Behaviour 3, 221-229 (2019).

[78] Brewer, M. B. The social self: On being the same and different at the same time. Personality and Social Psychology Bulletin 17, 475-482 (1991).

[79] Berger, J. \& Heath, C. Who drives divergence? identity signaling, outgroup dissimilarity, and the abandonment of cultural tastes. Journal of Personality and Social Psychology 95, 593 (2008).

[80] Krause, J., James, R. \& Croft, D. Personality in the context of social networks. Philosophical Transactions of the Royal Society B: Biological Sciences 365, 4099-4106 (2010).

[81] McPherson, M., Smith-Lovin, L. \& Cook, J. M. Birds of a feather: Homophily in social networks. Annual Review of Sociology 27, 415-444 (2001).

[82] Frankenhuis, W. E. \& Panchanathan, K. Balancing sampling and specialization: An adaptationist model of incremental development. Proceedings of the Royal Society of London B 278, 3558-3565 (2011).

[83] Panchanathan, K. \& Frankenhuis, W. E. The evolution of sensitive periods in a model of incremental development. Proceedings of the Royal Society of London B 283, 20152439 (2016).

[84] Frankenhuis, W. E., Panchanathan, K. \& Belsky, J. A mathematical model of the evolution of individual differences in developmental plasticity arising through parental bet-hedging. Developmental science 19, 251-274 (2016).

[85] Smaldino, P. E. \& Richerson, P. J. The origins of options. Frontiers in Neuroscience 6, 50 (2012).

[86] Heyes, C. Cognitive gadgets: The cultural evolution of thinking (Harvard University Press, 2018). 